\title{
Compactification of Gauge Theories and the Gauge Invariance of Massive Modes
}

\author{
R. Amorim and J. Barcelos-Neto \\ Instituto de Física \\ Universidade Federal do Rio de Janeiro \\ RJ 21945-970, Caixa Postal 68528, Brasil
}

Received on 13 December, 2001

\begin{abstract}
We study the gauge invariance of the massive modes in the compactification of gauge theories from $D=5$ to $D=4$. We deal with Abelian gauge theories of rank one and two, and with nonAbelian ones of rank one. We show that Stückelberg fields naturally appear in the compactification mechanism, contrarily to what usually occurs in literature where they are introduced by hand, as a trick, to render gauge invariance for massive theories. We also show that in the non-Abelian case they appear in a very different way when compared with their usual implementation in the non-Abelian Proca model.
\end{abstract}

\section{Introduction}

Nowadays, there is broad consensus that fundamental theories might come from spacetime dimension $D$ higher than four, probably $D=10$ or $D=11$. This is mainly related to the advent of string theories, that are consistent in the quantum word just at $D=10$. Further, the duality among known string theories suggests that they might emerge from a more fundamental theory at $D=11$. However, one of the great drawback of this idea is that there is no rule about some specific mechanisms to reach our word at $D=4$.

One manner of gaining some insight on this problem is to study the compactification close to $D=4$ and try to understand the features that a possible fundamental theory should have from the theoretical consistency of the results. It is important to mention that the seminal idea related to this point of view dates back a long time ago, in the works of Kaluza and Klein [1, 2], where they have started from the gravitational Einstein theory at $D=5$ and, after spontaneous compactification, reached the Maxwell and Einstein theories at $D=4$. The vector gauge field $A^{\mu}$ was originated from a component of the metric tensor. It is opportune to emphasize that many of the recent attempts to implement compactification mechanism have the Kaluza-Klein idea as a strong support.

We would like to address the present paper to this line. We consider the compactification of gauge theories of ranks one and two from $D=5$ to $D=4$ and study the question of gauge invariance in $D=4$, that shall be retained in the compactification procedure, even for the massive modes. We shall see that Stückelberg fields [3], that are usually introduced as a trick in order to attain the gauge invariance of massive vector fields, naturally emerge in this procedure. Even though we are going to deal just with gauge fields of rank one and two (that are the only ones whose number of physical degrees of freedom are consistent at $D=4$ ), the method can be directly extended for gauge fields of any rank at higher spacetime dimensions. Concerning to the non-Abelian case, we mention that the non-Abelian formulation of theories of rank higher than one cannot be directly done. The gauge invariance, even for the massless case, is only achieved by means of auxiliary fields [4]. We shall deal with the non-Abelian case just for rank one. We mention that the role played by the Stückelberg fields in this case is very different from their usual implementation in the non-Abelian Proca model.

Our work is organized as follows: In Section II we treat the spontaneous compactification of Maxwell theory originally defined in $D=5$, and show that in the Fourier expansion procedure, the zero modes correspond to the usual Maxwell and real massless scalar fields in $D=4$. The nonzero modes correspond to complex Proca fields coupled to the appropriate complex Stückelberg fields to keep the gauge invariance. In Section III we apply this procedure in the two-form Abelian gauge theory originally described in $D=5$. After compactification, we get Maxwell and massless 
two-form gauge theories for the zero modes. The vector fields play the role of Stückelberg fields for the massive modes of the two-form fields. In section IV, we consider Yang-Mills theory. Although it has the Abelian limit found in Section II, an interesting gauge structure is obtained in the full theory, where modes and gauge multiplets are mixed in a non-trivial way in order to keep the gauge invariance of the action. We reserve Section $\mathrm{V}$ for some concluding remarks.

\section{Maxwell theory}

Let us consider the following action for the Maxwell theory at $D=5$

$$
S=\frac{1}{R} \int d^{4} x \int_{0}^{R} d x^{4}\left(-\frac{1}{4} F^{M N} F_{M N}\right)
$$

where the coordinate $x^{4}$ describes a circle of radius $R$. We use capital Roman indices to express the spacetime dimension $D=5$, i.e. $M, N=0, \cdots, 4$, and adopt the metric convention $\eta^{M N}=\operatorname{diag}(+1,-1,-1,-1,-1)$. The Maxwell stress tensor $F^{M N}$ is defined in terms of the potential vector $A^{M}$ by the usual relation

$$
F^{M N}=\partial^{M} A^{N}-\partial^{N} A^{M}
$$

The action (2.1) is invariant under the gauge transformation

$$
\delta A^{M}=\partial^{M} \Lambda
$$

Let us split the vector potential $A^{M}$ as $A^{M}=A^{\mu}$ for $M=0, \cdots, 3$ and $A^{M}=\phi$ for $M=4$. We thus have for the action (2.1)

$$
\begin{array}{r}
S=\frac{1}{R} \int d^{4} x \int_{0}^{R} d x^{4}\left(-\frac{1}{4} F^{\mu \nu} F_{\mu \nu}-\frac{1}{2} \partial^{\mu} \phi \partial_{\mu} \phi\right. \\
\left.-\frac{1}{2} \partial^{4} A^{\mu} \partial_{4} A_{\mu}+\partial^{\mu} \phi \partial^{4} A_{\mu}\right)
\end{array}
$$

The first term above cannot be identified with the Maxwell Lagrangian at $D=4$ because $A^{\mu}$ depends on both $x^{\mu}$ and $x^{4}$. Following the usual procedure in the (spontaneous) compactification procedure [2], we take the expansions of $A^{\mu}$ and $\phi$ in Fourier harmonics, namely

$$
\begin{aligned}
A^{\mu}\left(x, x^{4}\right) & =\sum_{n=-\infty}^{+\infty} A_{(n)}^{\mu}(x) \exp \left(2 i n \pi \frac{x^{4}}{R}\right) \\
\phi\left(x, x^{4}\right) & =\sum_{n=-\infty}^{+\infty} \phi_{(n)}(x) \exp \left(2 i n \pi \frac{x^{4}}{R}\right)
\end{aligned}
$$

The replacement of these expansions into the action (2.4) leads to

$$
\begin{aligned}
S=\int d^{4} x & \sum_{n=-\infty}^{+\infty}\left(-\frac{1}{4} F_{(n)}^{\mu \nu} F_{(-n) \mu \nu}-\frac{1}{2} \partial^{\mu} \phi_{(n)} \partial_{\mu} \phi_{(-n)}\right. \\
& \left.-\frac{2 \pi^{2} n^{2}}{R^{2}} A_{(n)}^{\mu} A_{(-n) \mu}+\frac{2 i n \pi}{R} A_{(n)}^{\mu} \partial_{\mu} \phi_{(-n)}\right)
\end{aligned}
$$

Since $A_{M}$ is a real quantity, we have from expansions (2.5) that $A_{(-n)}^{\mu}=A_{(n)}^{* \mu}$ and $\phi_{(-n)}=\phi_{(n)}^{*}$. Using this into the action (2.6) we rewrite it in a more convenient way

$$
\begin{aligned}
S=\int d^{4} x\{ & -\frac{1}{4} F_{(0)}^{\mu \nu} F_{(0) \mu \nu}-\frac{1}{2} \partial^{\mu} \phi_{(0)} \partial_{\mu} \phi_{(0)} \\
& \left.+\sum_{n=1}^{\infty}\left[-\frac{1}{2} F_{(n)}^{\mu \nu} F_{(n) \mu \nu}^{*}-\frac{4 n^{2} \pi^{2}}{R^{2}}\left(A_{(n)}^{\mu}+\frac{i R}{2 n \pi} \partial^{\mu} \phi_{(n)}\right)\left(A_{(n) \mu}^{*}-\frac{i R}{2 n \pi} \partial_{\mu} \phi_{(n)}^{*}\right)\right]\right\}
\end{aligned}
$$

We observe that the two zero mode terms at $D=4$ correspond to Maxwell and real scalar field theories. The other modes are related to complex Proca fields with masses given by $2 n \pi / R$. It is interesting to note the role played by the corresponding modes of the scalar fields. They are Stückelberg fields. Usually, these are put by hand as a trick to make the Proca theory gauge invariant or to implement the Hamiltonian embedding procedure, during the conversion of second to first-class constraints [5]. Here, these fields naturally emerge in order to keep the gauge symmetry of the initial theory.

\section{Abelian two-form}

The natural extension of what was done in the previous section is to consider gauge fields of rank two. Let us consider this in the present section by starting from the action [6] 


$$
S=\frac{1}{12 R} \int d^{4} x \int_{0}^{R} d x^{4} H^{M N P} H_{M N P}
$$

The completely antisymmetric stress tensor $H^{M N P}$ is defined in terms of the antisymmetric two-form gauge field $B^{M N}$ by

$$
H^{M N P}=\partial^{M} B^{N P}+\partial^{P} B^{M N}+\partial^{N} B^{P M}
$$

This theory is invariant for the (reducible) [6, 7] gauge transformation

$$
\delta B^{M N}=\partial^{M} \Lambda^{N}-\partial^{N} \Lambda^{M}
$$

In order to perform the compactification to $D=4$, we conveniently split the potential $B^{M N}$ as

$$
\begin{aligned}
B^{M N} & =\left(B^{\mu \nu}, B^{4 \mu}\right) \\
& =\left(B^{\mu \nu}, A^{\mu}\right)
\end{aligned}
$$

where we have identified $B^{4 \mu}$ with $A^{\mu}$. Again, this is not the vector potential of the Maxwell theory because it depends on both $x^{\mu}$ and $x^{4}$ and its gauge transformation, according to (3.3), reads

$$
\delta A^{\mu}=\partial^{\mu} \Lambda^{4}-\partial^{4} \Lambda^{\mu}
$$

which is not the characteristic transformation of the Maxwell connection.

Introducing (3.4) into (3.1), we obtain

$$
\begin{aligned}
S=\frac{1}{R} \int d^{4} x & \int_{0}^{R} d x^{4}\left(\frac{1}{12} H^{\mu \nu \rho} H_{\mu \nu \rho}-\frac{1}{4} F^{\mu \nu} F_{\mu \nu}\right. \\
& \left.+\frac{1}{4} \partial^{4} B^{\mu \nu} \partial_{4} B_{\mu \nu}+\frac{1}{2} F^{\mu \nu} \partial^{4} B_{\mu \nu}\right)
\end{aligned}
$$

where, for the same previous argument, $F^{\mu \nu}=\partial^{\mu} A^{\nu}-$ $\partial^{\nu} A^{\mu}$ is not the Maxwell stress tensor. Expanding the fields $B^{\mu \nu}, A^{\mu}$, as well as the gauge parameters $\Lambda^{\mu}$ and $\Lambda^{4}$ in Fourier harmonics, we have

$$
\begin{aligned}
B^{\mu \nu}\left(x, x_{4}\right) & =\sum_{n=-\infty}^{+\infty} B_{(n)}^{\mu \nu}(x) \exp \left(2 i n \pi \frac{x^{4}}{R}\right) \\
A^{\mu}\left(x, x_{4}\right) & =\sum_{n=-\infty}^{+\infty} A_{(n)}^{\mu}(x) \exp \left(2 i n \pi \frac{x^{4}}{R}\right) \\
\Lambda^{\mu}\left(x, x_{4}\right) & =\sum_{n=-\infty}^{+\infty} \xi_{(n)}^{\mu}(x) \exp \left(2 i n \pi \frac{x^{4}}{R}\right) \\
\Lambda^{4}\left(x, x_{4}\right) & =\sum_{n=-\infty}^{+\infty} \xi_{(n)}(x) \exp \left(2 i n \pi \frac{x^{4}}{R}\right)
\end{aligned}
$$

Introducing these quantities into (3.6) and considering that $B_{(-n)}^{\mu \nu}=B_{(n)}^{* \mu \nu}, A_{(-n)}^{\mu}=A_{(n)}^{* \mu}$, etc., we obtain

$$
\begin{aligned}
S=\int d^{4} x[ & \frac{1}{12} H_{(0)}^{\mu \nu \rho} H_{(0) \mu \nu \rho}^{*}-\frac{1}{4} F_{(0)}^{\mu \nu} F_{(0) \mu \nu}^{*} \\
& +\sum_{n=1}^{\infty}\left(\frac{1}{6} H_{(n)}^{\mu \nu \rho} H_{(n) \mu \nu \rho}^{*}-\frac{1}{2} F_{(n)}^{\mu \nu} F_{(n) \mu \nu}^{*}\right. \\
& \left.\left.+\frac{2 n^{2} \pi^{2}}{R^{2}} B_{(n)}^{\mu \nu} B_{(n) \mu \nu}^{*}-\frac{2 i n \pi}{R} F_{(n)}^{\mu \nu} B_{(n) \mu \nu}^{*}\right)\right]
\end{aligned}
$$

Due to the gauge transformations of the zero mode fields,

$$
\begin{aligned}
& \delta A_{(0)}^{\mu}=\partial^{\mu} \xi_{(0)} \\
& \delta B_{(0)}^{\mu \nu}=\partial^{\mu} \xi_{(0)}^{\nu}-\partial^{\nu} \xi_{(0)}^{\mu}
\end{aligned}
$$

we have that the two zero mode terms of (3.8) are the tensor and vector (Maxwell) theories at $D=4$ [8]. The remaining modes correspond to massive complex tensor gauge fields $B_{(n)}^{\mu \nu}$ and massless vector ones $A_{(n)}^{\mu}$. Let us rewrite the $n$-mode terms of expression (3.8) in a more appropriate form

$$
S_{(n)}=\int d^{4} x\left[\frac{1}{6} H_{(n)}^{\mu \nu \rho} H_{(n) \mu \nu \rho}^{*}-\frac{2 n^{2} \pi^{2}}{R^{2}}\left(i B_{(n)}^{\mu \nu}-\frac{R}{2 n \pi} F_{(n)}^{\mu \nu}\right)\left(-i B_{(n) \mu \nu}^{*}-\frac{R}{2 n \pi} F_{(n) \mu \nu}^{*}\right)\right]
$$

We notice that in the rank 2 theory, the massless vector gauge field $A_{(n)}^{\mu}$ naturally appears as a Stückelberg field for the massive antisymmetric gauge field $i B_{(n)}^{\mu \nu}$. Their gauge transformations are given by

$$
\begin{aligned}
& \delta A_{(n)}^{\mu}=\partial^{\mu} \xi_{(n)}-\frac{2 i \pi n}{R} \xi_{(n)}^{\mu} \\
& \delta B_{(n)}^{\mu \nu}=\partial^{\mu} \xi_{(n)}^{\nu}-\partial^{\nu} \xi_{(n)}^{\mu}
\end{aligned}
$$

We mention that a similar result, where vector fields play the role of Stückelberg fields for the massive rank two theory, was also found in the case of Hamiltonian embedding mechanism [9].

We could generalize this analysis for gauge fields of any rank. However, for $D=4$ it does not make sense to consider gauge fields of rank higher than two, because the gauge and redutibility conditions would lead to a negative number of physical degrees of freedom. 


\section{Non-Abelian case}

As already said, the non-Abelian formulation of gauge theories with rank two or higher cannot be directly implemented. Its gauge invariance can only be achieved with the help of auxiliary fields [4]. Consequently, since these models do not have a clear gauge invariance, their discussion here will be avoided. We shall only consider in this section the vector case. The non-Abelian version of the procedure described in section II should start from the action

$$
S=\frac{1}{R} \int d^{4} x \int_{0}^{R} d x^{4} \operatorname{tr}\left(-\frac{1}{4} F^{M N} F_{M N}\right)
$$

where now

$$
F^{M N}=\partial^{M} A^{N}-\partial^{N} A^{M}-i\left[A^{M}, A^{N}\right]
$$

and the gauge potentials take values in a $S U(N)$ algebra, whose hermitian generators are assumed to satisfy a normalized trace condition. The action (4.1) is invariant under the gauge transformation

$$
\delta A^{M}=D^{M} \Lambda
$$

once one defines the covariant derivative as

$$
D^{M} \Lambda=\partial^{M} \Lambda-i\left[\Lambda, A^{M}\right]
$$

Again writing $A^{4}=\phi$, we note that $F^{4 \mu}=\partial^{4} A^{\mu}-D^{\mu} \phi$ permits to rewrite action (4.1) as

$$
\begin{aligned}
S=\frac{1}{R} \int & d^{4} x \int_{0}^{R} d x^{4} \operatorname{tr}\left(-\frac{1}{4} F^{\mu \nu} F_{\mu \nu}\right. \\
& \left.-\frac{1}{2} D^{\mu} \phi D_{\mu} \phi-\frac{1}{2} \partial^{4} A^{\mu} \partial_{4} A_{\mu}+D^{\mu} \phi \partial^{4} A_{\mu}\right)
\end{aligned}
$$

Expanding the fields above in Fourier harmonics in a similar way to Sec. II, we get

$$
S=\int d^{4} x \operatorname{tr} \sum_{n=-\infty}^{\infty}\left[-\frac{1}{4} F_{(n)}^{\mu \nu} F_{(-n) \mu \nu}-\frac{1}{2}\left(\frac{2 n \pi}{R} A_{(n)}^{\mu}+i D^{\mu} \phi_{(n)}\right)\left(\frac{2 n \pi}{R} A_{(-n) \mu}-i D_{\mu} \phi_{(-n)}\right)\right]
$$

where

$$
\begin{aligned}
& \left(D^{\mu} \phi\right)_{(n)}=\partial^{\mu} \phi_{(n)}+i \sum_{m=-\infty}^{+\infty}\left[\phi_{(m)}, A_{(n-m)}^{\mu}\right] \\
& F_{(n)}^{\mu \nu}=\partial^{\mu} A_{(n)}^{\nu}-\partial^{\nu} A_{(n)}^{\mu}-i \sum_{m=-\infty}^{+\infty}\left[A_{(m)}^{\mu}, A_{(n-m)}^{\nu}\right]
\end{aligned}
$$

It is interesting to observe that the non-Abelian character of the action (4.6) induces a gauge structure where the modes mixed among themselves. In other words, the gauge multiplet and the modes form a nontrivial structure that has to be considered as a whole to preserve the symmetry of the action. Actually, the transformations (4.3) have their mode expanded version given by

$$
\begin{aligned}
& \delta A_{(n)}^{\mu}=\left(D^{\mu} \Lambda\right)_{(n)} \\
& \delta \phi_{(n)}=\frac{i 2 n \pi}{R} \Lambda_{(n)}-i \sum_{m=-\infty}^{+\infty}\left[\Lambda_{(m)}, A_{(n-m)}^{\mu}\right]
\end{aligned}
$$

where the covariant derivative is defined in (4.7). As a consequence of the above equations,

$$
\begin{aligned}
& \delta F_{(n)}^{\mu \nu}=-i \sum_{m=-\infty}^{+\infty}\left[\Lambda_{(m)}, F_{(n-m)}^{\mu \nu}\right] \\
& \delta\left(\frac{2 n \pi}{R} A_{(n)}^{\mu}+i\left(D^{\mu} \phi\right)_{(n)}\right)=-i \sum_{m=-\infty}^{+\infty}\left[\Lambda_{(m)}, \frac{2(n-m) \pi}{R} A_{(n-m)}^{\mu}+i\left(D^{\mu} \phi\right)_{(n-m)}\right]
\end{aligned}
$$


which is a symmetry of action (4.6), as can be verified. It is interesting to observe that the role played by the Stückelberg fields here is different from the usual one presented by the non- Abelian Proca model [10]. There, the Stückelberg field is introduced by hand in order to just give $\delta\left[m A^{\mu}+i\left(D^{\mu} \phi\right)\right]=0$.

\section{Conclusion}

In this paper we have considered the spontaneous compactifications of Maxwell, Abelian two-form, and nonAbelian one-form gauge theories from a $D=5$ spacetime with a compact dimension to the usual $D=4$ Minkowski spacetime. We have focused our attention to the gauge invariance of the theories formulated at $D=5$, which should be kept along the compactification procedure. As usual, there arise massive modes in the process of compactification. In principle, the gauge invariance of these modes could be lost. We observe, however, that generalized Stückelberg fields naturally emerge in order to keep the content of the original gauge invariance. Although in the Abelian cases the Stückelberg fields correspond to those already found in the literature, the compactification of the Yang-Mills theory reveals a new structure of compensating fields. Because of the nonlinearity of the action, the gauge structure displayed by the mode expansion of covariant derivatives, curvature tensors and gauge transformations play a remarkable feature in mixing Fourier modes and gauge multiplet components in a nontrivial way.

Acknowledgment: This work is supported in part by Conselho Nacional de Desenvolvimento Científico e Tecnológico - CNPq (Brazilian Research agency) with the support of PRONEX 66.2002/1998-9.

\section{References}

[1] T. Kaluza, Akad. Wiss. Phys. Math. K1, 966 (1921); O. Klein, Z. Phys. 37, 895 (1926).

[2] For a more general reference on compactification mechanism, see for example, L. Castellani, R. D'Auria and P. Fré, Supergravity and Superstrings - A Geometric Perspective (World Scientific, 1991), and references therein.

[3] E.C.G. Stückelberg, Helv. Phys. Acta 30, 209 (1957).

[4] A. Larihi, Phys. Rev. D55, 5045 (1997); D.S. Hwang and C.-Y Lee, J. Math. Phys. 38, 30 (1997); J. Barcelos-Neto, A. Cabo and M.B.D. Silva, Z. Phys. C72, 345 (1996).

[5] I.A. Batalin and E.S. Fradkin, Phys. Lett. B180, 157 (1986); Nucl. Phys. B279, 514 (1987); I.A. Batalin, E.S. Fradkin, and T.E. Fradkina, ibid. B314, 158 (1989); B323, 734 (1989); I.A. Batalin and I.V. Tyutin, Int. J. Mod. Phys. A6, 3255 (1991).

[6] M. Kalb and P. Ramond, Phys. Rev. D9, 2273 (1974).

[7] For a review see M. Henneaux, Phys. Rep. C126, 1 (1985). A more complete perspective can be found in M. Henneaux and C. Teitelboim, Quantization of gauge systems (Princeton Univ. Press, 1992).

[8] J. Barcelos-Neto, J. Math. Phys. 41, 6661 (2000).

[9] C. Bizdadea and S.O. Saliu, Phys. Lett. B368, 202 (1996).

[10] T. Kunimasa and T. Goto, Prog. Theor. Phys. 37, 452 (1967). 